\title{
Elastosis perforans serpiginosa: a review of the literature and our own experience
}

\author{
Adriana Polańska ${ }^{1}$, Monika Bowszyc-Dmochowska², Ryszard W. Żaba ${ }^{1}$, Zygmunt Adamski ${ }^{3}$, Adam Reich $^{4}$, \\ Aleksandra Dańczak-Pazdrowska ${ }^{3}$ \\ ${ }^{1}$ Department of Dermatology and Venereology, Poznan University of Medical Sciences, Poznan, Poland \\ ${ }^{2}$ Cutaneous Histopathology and Immunopathology Section, Department of Dermatology, Poznan University of Medical Sciences, \\ Poznan, Poland \\ ${ }^{3}$ Department of Dermatology, Poznan University of Medical Sciences, Poznan, Poland \\ ${ }^{4}$ Department of Dermatology, Venereology and Allergology, Wroclaw Medical University, Wroclaw, Poland
}

Adv Dermatol Allergol 2016; XXXIII (5): 392-395

DOI: $10.5114 / a d a .2016 .62849$

Elastosis perforans serpiginosa (EPS) is a rare disorder classified as a primary perforating dermatosis. That group of diseases also includes reactive perforating collagenosis, perforating folliculitis or Kyrle disease. In EPS, protrusions of altered elastic fibers and other connective tissue material through the papillary layer and epidermis (called transepithelial elimination) clinically manifest as small papules arranged in an annular pattern [1, 2].

The first description of EPS was most likely published in 1927 (Fisher), and at that time, because of the clinical presentation, this condition was classified into a group of diseases with hyperkeratosis. In 1953, Lutz described the morphology of EPS and named it serpiginosa follicular keratosis. In 1955, Miescher showed that the extruded material contains elastin and suggested the term elastomer intrapapillare perforans veruciforme, which in 1958, Dammert and Putkonem changed into EPS [1, 2].

Elastosis perforans serpiginosa is considered as mild dermatosis, which is not accompanied by involvement of other organs [2]. The disease prevalence has not been clearly specified so far but this is not a condition commonly found in routine clinical practice. It is believed that $90 \%$ of EPS cases are patients before the age of 30 , although 5 -year-old and 89-year-old patients have been reported in the literature. Elastosis perforans serpiginosa more often affects men and only $25 \%$ of cases were detected among women [2, 3].

In the course of EPS, the presence of erythematous as well as skin-colored papules or plaques with keratotic surface which are grouped together and form serpiginous or annular lesions is usually observed. The lesions tend to subside in the center and often solitary satellite lesions in the close vicinity are detected. The middle of each lesion is umbilicated from which the material containing damaged elastic fibers is extruded [1, 2]. With the exception of EPS occurring in the course of Down's syndrome and penicillamine treatment, skin lesions are usually symmetrical. In addition, they are mostly single, and the presence of numerous scattered plaques is atypical. The lesions are mostly located on the arms, face and neck. There is a slow course of the disease and in some cases a spontaneous regression of lesions in the period from 6 months to 5 years with a possibility of scarring may appear [1-4]. Figures $1 \mathrm{~A}-\mathrm{C}$ present typical clinical presentation of EPS.

We can distinguish three subtypes of EPS. The first one, known as reactive one, is associated with connective tissue diseases, such as Ehlers-Danlos syndrome (type IV), cutis laxa, Marfan syndrome, acrogeria, Rothmund-Thomson syndrome, osteogenesis imperfecta and pseudoxanthoma elasticum [3-6]. A list of possible comorbidities of EPS is shown in Table 1 [3-6]. The relationship between EPS and Down's syndrome is unclear, but some symptoms, such as premature aging or excessive flexibility of joints may suggest a link to exist $[3,4]$. The second form of the disease is a drug-induced one, which is caused by treatment with D-penicillamine. In this type of EPS, the disease occurrence is observed in the first year of treatment and it is estimated that EPS may affect about $1 \%$ of patients who underwent such therapy [4, 7]. The last subtype, idiopathic one, may be associated with genetic basis of the autosomal dominant type of

Address for correspondence: Adriana Polańska MD, PhD, Department of Dermatology and Venereology, Poznan University of Medical Sciences, 49 Przybyszewskiego St, 60-355 Poznan, Poland, phone: +48 516123 858, e-mail: adriana-polanska@wp.pl Received: 15.01.2016, accepted: 29.02.2016. 

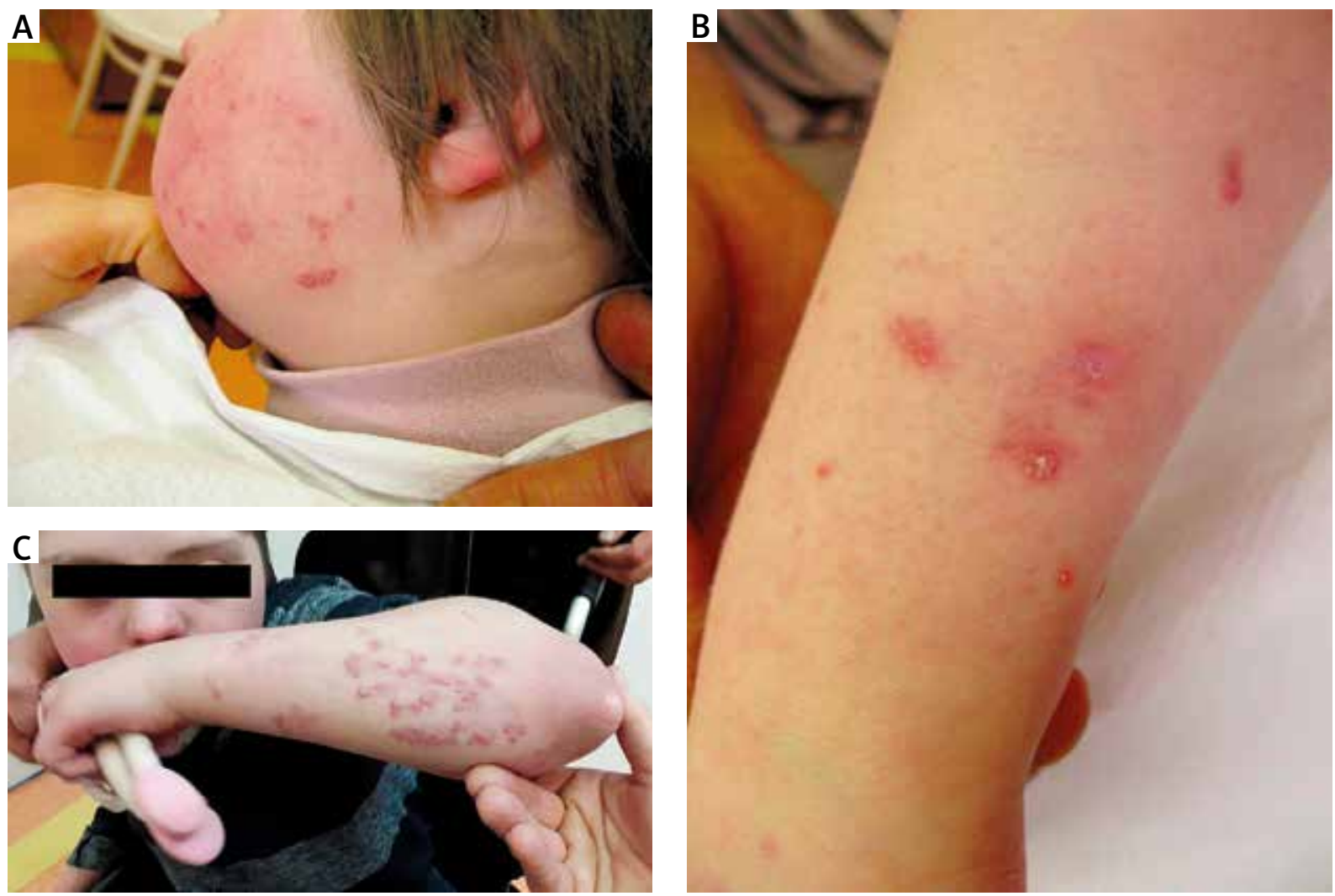

Figure 1. Characteristic for EPS skin lesions within face (A) and left upper extremity (B, C) - papular keratotic lesions fuced together in serpiginous arrangement

inheritance. However, to date, the candidate gene has not been identified [6, 8].

Etiology and pathogenesis of EPS has not yet been fully understood. It is believed that the local skin inflammation induces formation of perifollicular or intraepithelial tunnels through which abnormal elastic fibers are secreted [2]. In 2000, Fujimoto et al. revealed the presence of $67 \mathrm{kDa}$ elastin receptor peptides on keratinocytes near the transepidermal tunnel, which probably allows the fiber migration and other phenomena of EPS changes $[9,10]$.

The diagnosis of EPS should not be based solely on the dermatological examination and evaluation of EPS-specific skin lesions, but rather on the histological assessment of biopsy of the affected skin [1]. In the biopsies an increased amount of elastic fibers can be found, both just underneath the epidermis and in the reticular layer of the dermis. The fibers are compacted, twisted and fragmented, what can be clearly seen in the staining for elastic fibers such as in van Gieson staining. The fibers in the papillary layer are thickened, arranged vertically and penetrate into the skin, where they form characteristic narrow tunnels with a right, wave or spiral arrangement. In the transepidermal channel, and par-
Table 1. Diseases associated with elastosis perforans serpiginosa

\begin{tabular}{l}
\hline Acrogeria \\
\hline Ehlers-Danlos syndrome \\
\hline Marfan syndrome \\
\hline Morphea \\
\hline Osteogenesis imperfecta \\
\hline Pseudoxanthoma elasticum \\
\hline Rothmund-Thomson syndrome \\
\hline Cutis laxa
\end{tabular}

ticularly in its lower part, basophilic degeneration mass consisting of keratinocytes, remainings of crumbled inflammatory cells and elastic fibers can be detected. The channel opens into a bowl-shaped cavity filled with horny plug. Partially the parakeratotic keratin plug contains an addition of basophilic masses and crumbled nuclei of inflammatory cells. The epidermis in the vicinity of the channel is thicker and covers a tunnel in the shape of pincers. The skin at the entrance of the tunnel accumulates chronic inflammatory infiltrate composed often of giant cells $[11,12]$. This characteristic histopathologic picture 

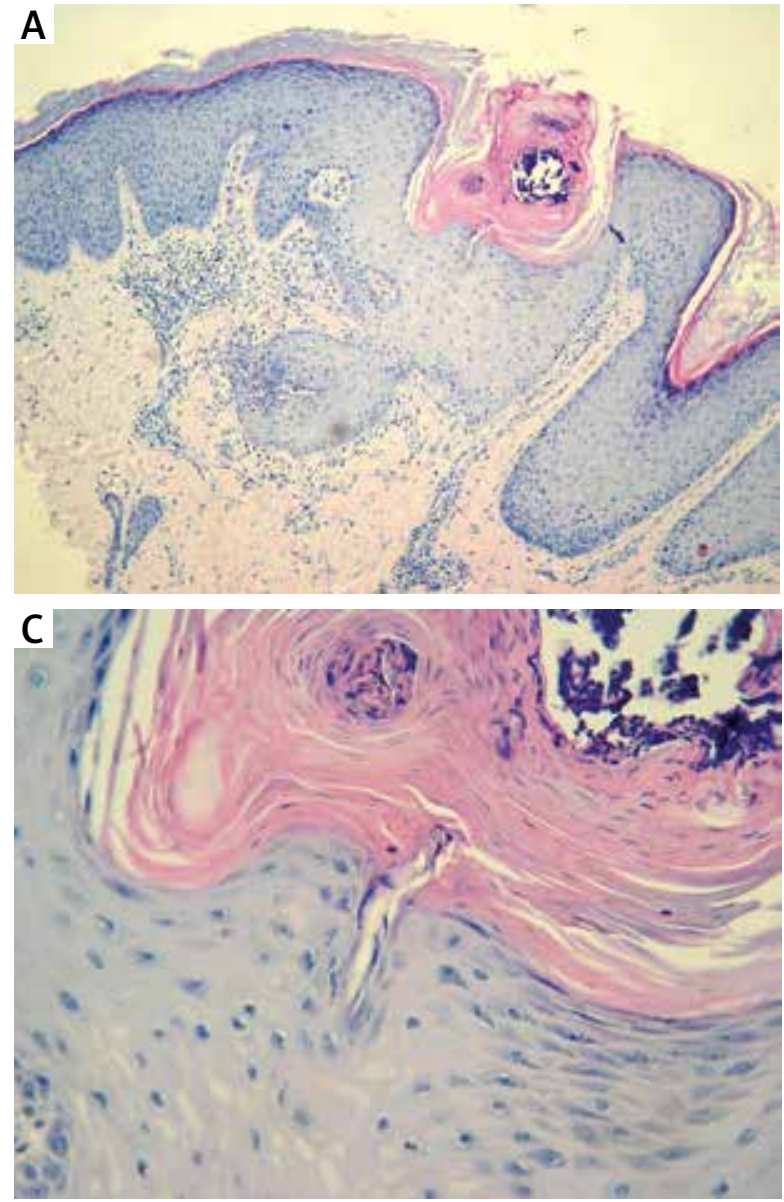

of EPS is presented in Figures $2 \mathrm{~A}-\mathrm{C}[1-3]$. In cases of penicillamine-induced EPS, calcification can be observed between the collagen fibers [1-4].

Due to its rarity, EPS often remains unrecognized, but the diagnosis should always be taken into account in children, in whom annular lesions on the face and neck can be visualized. The differential diagnosis should also include granuloma annulare, dermatophyte infections, sarcoidosis, skin calcinosis and porokeratosis of Mibelli [1].

There is no effective standard treatment of EPS, and so far there have been only single reports of the use of different drugs for systemic and topical therapy [2]. Outland et al. presented successful treatment with $0.1 \%$ tazarotene gel, and Kelly and Purcell indicated the reduction of skin after 10-week application of imiquimod [13, 14]. There are also conflicting reports about the positive therapeutic effect of laser therapy, including $\mathrm{CO}_{2}$ laser, Er: YAG laser and pulsed dye lasers. Some researchers have demonstrated the effectiveness of the laser light, like Abdullah et al. who observed 18-month remission after aggressive sessions with $\mathrm{CO}_{2}$ laser [15]. However, others, like Saxena did not show a positive response to numerous sessions using these lasers, and in turn, $\mathrm{CO}_{2}$

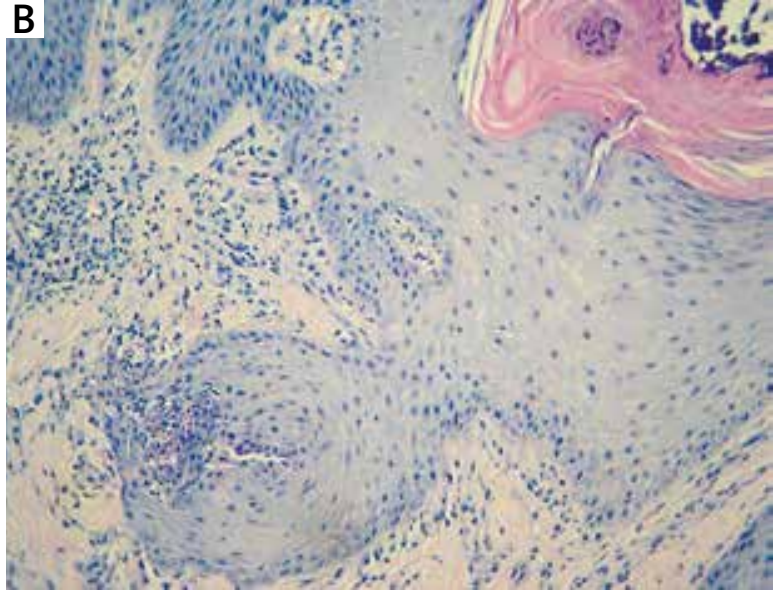

Figure 2. Histopathologic picture of EPS with thick epidermis and transepidermal channel $(\mathbf{A}-\mathbf{C})$, horny plug $(\mathbf{A}, \mathbf{C})$ and mixed inflammatory infiltration within the dermis (B)

laser caused scarring [16]. Moreover, attempts to treat EPS with oral isotretinoin, local corticosteroid injections, electrocoagulation, cryotherapy as well as UVB irradiation were performed but the effect was poor [3, 4]. In the first case, initially they unsuccessfully used 3-month therapy with tretinoin cream, then cryotherapy was performed, yielding only a minimal improvement. In the second patient they observed a slight reduction in lesions after simultaneous treatment with $0.1 \%$ tazarotene cream (2 times daily) and local cryotherapy. In the latter case, the treatment has not been undertaken [6]. The latest report of Chinese authors suggests the effective treatment of photodynamic therapy (PDT) with 5-aminolevulinic acid (ALA) in penicillamine-induced refractory EPS [17].

We present a 16-year-old girl with Down's syndrome and plaque psoriasis in whom we diagnosed EPS. In addition, the patient was suffering from hypothyroidism, tetralogy of Fallot and bilateral congenital dislocation of the hip joints. The dermatological examination revealed some reduced psoriatic plaques on the skin of the elbows and scalp. Exfoliating erythematous papular lesions with a diameter of a few millimeters within the skin of the left cheek and upper and lower limbs were observed. 
The lesions were fusing into an annular arrangement, spreading outwards and leaving erythematous macule in the center (Figures $1 \mathrm{~A}, \mathrm{~B}$ ). According to the child's mother, these lesions were not pruritic, however the mother did not deny manipulation within lesions by her child.

To confirm the clinical diagnosis, the biopsy was taken from the primary circinate lesion of the cheek. The histopathologic examination revealed the thick epidermis, typical of EPS, and a transepithelial narrow channel of a wavy configuration (Figure $2 \mathrm{~A}$ ). After obtaining the result of the histopathological examination, the nature of the disease, possibilities as well as the effectiveness and purpose of the treatment were explained to the parents. The parents did not agree to cryotherapy in fear of scarring and also rejected topical therapy besides the use of $10 \%$ urea in ointment.

During next follow-up visits (the last one after 18 months from the first visit, Figure $1 \mathrm{C}$ ) we could detect an increase in the number of skin lesions typical of EPS with a linear arrangement, especially on the left upper extremity. We suspected that formation of new lesions could be induced by scratching and patient's manipulations and may be associated with Koebner phenomenon. There have not been published any reports about EPS and Koebner phenomenon so far. In this case, EPS coexisted with plaque psoriasis, however manipulations did not provoke psoriatic papules in this region, while evoked EPS lesions.

To the best of our knowledge, in the literature a similar case of simultaneous presence of EPS, Down's syndrome and psoriasis has not yet been reported. There have been previous data considering more frequent occurrence of psoriasis in patients with Down's syndrome (up to $8 \%$ ) than in the healthy population [18]. Furthermore, in this group of patients seborrhoeic dermatitis, skin dryness or the presence of hand and feet hyperkeratosis are the most common skin comorbidities. The association of psoriasis with Down's syndrome is still the subject of scientific discussion, and most studies show a possible disturbance in the interferon- $\gamma$ level (which is higher in those patients) [19].

\section{Conflict of interest}

The authors declare no conflict of interest.

\section{References}

1. de Rezende LN, Nuńez MG, Clavery TG, et al. Elastosis perforans serpiginosa. Indian Dermatol Online J 2014; 5: 236-7.

2. Pereira AC, Baeta IG, Costa Júnior SR, et al. Elastosis perforans serpiginosa in a patient with Down's syndrome. An Bras Dermatol 2010; 85: 691-4.

3. Lewis KG, Bercovitch L, Dill SW, Robinson-Bostom L. Acquired disorders of elastic tissue: part I. Increased elastic tissue and solar elastotic syndromes. J Am Acad Dermatol 2004; 51: 1-21.
4. Mehta RK, Burrows NP, Payne CM, et al. Elastosis perforans serpiginosa and associated disorders. Clin Exp Dermatol 2001; 26: 521-4.

5. Lee SH, Choi Y, Kim SC. Elastosis perforans serpiginosa. Ann Dermatol 2014; 26: 103-6.

6. Vearrier D, Buka RL, Roberts B, et al. What is standard of care in the evaluation of elastosis perforans serpiginosa? A survey of pediatric dermatologists. Pediatr Dermatol 2006; 23: $219-24$

7. Ishak R, Abbas O. Penicillamine revisited: historic overview and review of the clinical uses and cutaneous adverse effects. Am J Clin Dermatol 2013; 14: 223-33.

8. Rios-Buceta L, Amigo-Echenagusia A, Sols-Candelas M, et al. Elastosis perforans serpiginosa with simultaneous onset in two sisters. Int J Dermatol 1993; 32: 879-81.

9. Fujimoto N, Akagi A, Tajima S, et al. Expression of the 67$\mathrm{kDa}$ elastin receptor in perforating skin disorders. Br J Dermatol 2002; 146: 74-9.

10. Fujimoto N, Tajima S, Ishibashi A. Elastin peptides induce migration and terminal differentiation of cultured keratinocytes via $67 \mathrm{kDa}$ elastin receptor in vitro: $67 \mathrm{kDa}$ elastin receptor is expressed in the keratinocytes eliminating elastic materials in elastosis perforans serpiginosa. J Invest Dermatol 2000; 115: 633-9.

11. Lever WF, Schaumburg-Lever G. Histopathology of the Skin. $7^{\text {th }}$ ed. J.B. Lippincott Company, Philadelphia 1990.

12. Eduardo Calonje J, Brenn T, Lazar AJ, McKee PH. McKee's Pathology of the Skin. $4^{\text {th }}$ ed. Elsevier Saunders, Philadelphia 2011.

13. Outland JD, Brown TS, Callen JP. Tazarotene is an effective therapy for elastosis perforans serpiginosa. Arch Dermatol 2002; 138: 169-71.

14. Kelly SC, Purcell SM. Imiquimod therapy for elastosis perforans serpiginosa. Arch Dermatol 2006; 142: 829-30.

15. Abdullah A, Colloby PS, Foulds IS, Whitcroft I. Localized idiopathic elastosis perforans serpiginosa effectively treated by the Coherent Ultrapulse 5000 C aesthetic laser. Int J Dermatol 2000; 9: 719-20.

16. Saxena M, Tope WD. Response of elastosis perforans serpiginosa to pulsed CO2, Er:YAG, and dye lasers. Dermatol Surg 2003; 29: 677-8.

17. Wang D, Liang J, Xu J, Chen L. Effective treatment of d-penicillamine induced elastosis perforans serpiginosa with ALAPDT. Photodiagnosis Photodyn Ther 2015; 12: 140-2.

18. Madan V. Dermatological manifestations of Down's syndrome. Clin Exp Dermatol 2006; 31: 623-9.

19. Marmon S, De Souza A, Strober BE. Psoriasis and Down syndrome: a report of three cases and a potential pathophysiologic link. Dermatol Online J 2012; 18: 13. 